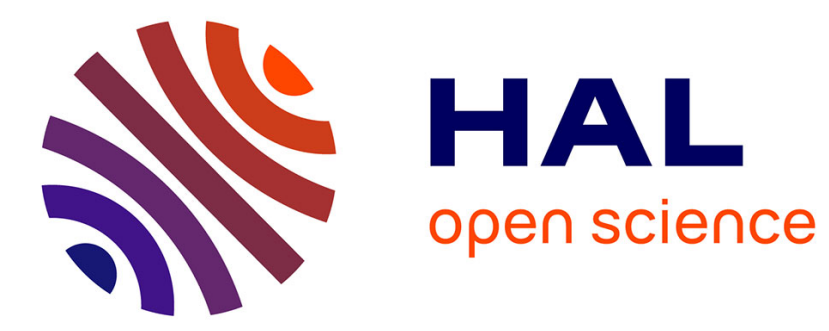

\title{
SRP-27 is a novel component of the supramolecular signaling complex involved in skeletal muscle excitation-contraction coupling
}

Christophe Bleunven, Susan Treves, Jinyu Xia, Elisa Leo, Michel Ronjat, Michel de Waard, Georg Kern, Bernhard E. Flucher, Francesco Zorzato

\section{To cite this version:}

Christophe Bleunven, Susan Treves, Jinyu Xia, Elisa Leo, Michel Ronjat, et al.. SRP-27 is a novel component of the supramolecular signaling complex involved in skeletal muscle excitation-contraction coupling. Biochemical Journal, 2008, 411 (2), pp.343-349. 10.1042/BJ20070906 . hal-00478843

\section{HAL Id: hal-00478843 https://hal.science/hal-00478843}

Submitted on 30 Apr 2010

HAL is a multi-disciplinary open access archive for the deposit and dissemination of scientific research documents, whether they are published or not. The documents may come from teaching and research institutions in France or abroad, or from public or private research centers.
L'archive ouverte pluridisciplinaire HAL, est destinée au dépôt et à la diffusion de documents scientifiques de niveau recherche, publiés ou non, émanant des établissements d'enseignement et de recherche français ou étrangers, des laboratoires publics ou privés. 
SRP-27 is a novel component of the supramolecular signaling complex involved in skeletal muscle excitation-contraction coupling.

Christophe Bleunven ${ }^{*}$, Susan Treves ${ }^{* \dagger}$, Jinyu Xia ${ }^{\dagger}$, Elisa Leo*, Michel Ronjat ${ }^{ \pm}$, Michel De Waard $^{ \pm}$, Georg Kern ${ }^{\S}$, Bernhard E. Flucher ${ }^{\S}$ and Francesco Zorzato ${ }^{* \dagger}$

*Department of Experimental and Diagnostic Medicine, General Pathology section, University of Ferrara, Via Borsari 46, 44100 Ferrara, Italy

†Departments of Anesthesia and Research, ZLF Basel University Hospital, Basel, Hebelstrasse 20, 4031 Basel, Switzerland

\pm Laboratoire Canaux Calciques, iRTSV/CCFP CEA Grenoble INSERM U836 Institut des Neurosciences Grenoble GIN, 17 rue des Martyrs, 38054 Grenoble Cedex 09, France.

$\S$ Department of Physiology and Medical Physics, Medical University Innsbruck, Fritz-Pregl-Str. 3, A-6020 Innsbruck, Austria.

To whom correspondence should be addressed:

Prof. F. Zorzato

Department of Experimental and Diagnostic Medicine

University of Ferrara, Via Borsari 46, 44100 Ferrara, Italy

email zor@unife.it

Running title: SRP-27, a novel $27 \mathrm{kDa}$ sarcoplasmic reticulum protein. 


\section{Summary}

SRP-27 is a newly identified integral membrane protein constituent of the skeletal muscle sarcoplasmic reticulum We identified its primary structure from cDNA clones isolated from a mouse skeletal muscle cDNA library. ESTs of SRP-27 were found mainly in cDNA libraries from excitable tissues of mouse. Western blot analysis confirmed the expression of SRP-27 in skeletal muscle and to a lower extent in heart and brain. Mild trypsin proteolysis combined with primary structure prediction analysis, suggest that SRP-27 has 4 transmembrane spanning alpha helices and its $\mathrm{COOH}$-terminal domain faces the cytoplasmic side of the endo(sarco)plasmic reticulum. The expression of SRP-27 is higher in fast twitch skeletal muscles compared to slow twitch muscles and peaks during the first month of post-natal development. High resolution immunohistochemistry and western blot analysis of subcellular fractions indicate that SRP-27 is distributed in both longitudinal tubules and in terminal cisternae of the sarcoplasmic reticulum, as well as in the perinuclear membrane systems and the nuclear envelope of myotubes and adult fibers. SRP-27 co-sediments with the ryanodine receptor macromolecular complex in high salt sucrose gradient centrifugation, is pulled-down by anti-ryanodine receptor antibodies as well as by maurocalcin, a well characterized ryanodine receptor modulator. Our data indicate that SRP-27 is part of a sarcoplasmic reticulum supramolecular complex suggesting the involvement of SRP-27 in the structural organization or function of the molecular machinery underlying excitation-contraction coupling (226 words). 


\section{Introduction}

The skeletal muscle sarcoplasmic reticulum (SR) is an intracellular membrane compartment that controls the myoplasmic calcium concentration and therefore plays a crucial role in excitationcontraction (E-C) coupling [1-3]. E-C coupling occurs in the triad, an intracellular junction formed by the association of the transverse tubules and the SR terminal cisternae [2-4]. E-C coupling is initiated by conformational changes of the L-type voltage dependent $\mathrm{Ca}^{2+}$ channel (DHPR), the voltage sensor localised within the membrane of the $\mathrm{T}$ tubular system [4,5]. Activation of $\mathrm{Ca}^{2+}$ release from SR terminal cisternae is due to the direct transmission of conformational changes of DHPR to ryanodine receptors (RyR). This model implies a close association between the SR terminal cisternae and T tubular membrane, and as well as the two channels, DHPR and RyR [5-10]. Detailed analysis of highly purified triad membrane fractions revealed that their protein composition is quite complex. The known protein components are: the $\mathrm{SR} \mathrm{Ca}^{2+}$ release channel (RyR), the DHPR, the triadin, the histidine rich $\mathrm{Ca}^{2+}$ binding protein (HRCP), the $90 \mathrm{kDa}$ JFP, calsequestrin, mitsugumins, junctophilin, JP45, junctate, the $32 \mathrm{kDa}$ ADT/ATP carrier [11-21], and a number of other polypeptides corresponding to still unidentified proteins. Because of their localisation in the junctional sarcoplasmic reticulum membrane, these proteins are likely to play a role either in the organization of the triad during development or in the mechanism of signal transduction in skeletal muscle. In this study, we discovered a $27 \mathrm{kDa}$ protein that we named SRP-27 (for sarcoplasmic reticulum protein-27 $\mathrm{kDa}$ ). We show that this polypeptide is present in skeletal muscle sarcoplasmic reticulum membranes as well as in the nuclear/endo(sarco)plasmic reticulum membrane of excitable tissues including heart and brain. SRP-27 co-sediments with the RyR complex, and its level of expression depends on fiber type differentiation and on the development stage. 


\section{Experimental Procedures}

\section{Materials}

Protein G-Sepharose, PCR primers, Peroxidase conjugated protein A, Protein-A Sepharose were from Sigma/Fluka; The pGEX-5X-3 plasmid, $\left[{ }^{35} \mathrm{~S}\right]$-methionine and Protein-A Sepharose were from Amersham Biosciences. The pEYFP-N1 plasmid was from Clontech. Nitrocellulose membrane was from Schleicher \& Schuell BioScience GmbH. Protein Molecular Weight markers were from Biorad. All other chemicals were reagent or highest available grade. Rats (Wistar) were bred in house. Rabbits (NZW) were purchased from Charles Rivers Laboratories (Calco, Italy). All procedures were performed in accordance with the stipulations of the Helsinki Declarations for care and use of laboratory animals.

\section{Methods}

\section{SRP-27 cDNA cloning}

Total RNA was isolated from rat limb skeletal muscles using Tri-Reagent (Molecular Research Center Inc.) following the instructions provided by the manufacturer. Total RNA was converted to cDNA using the cDNA Synthesis Kit from Roche Applied Science using a T17adapter primer 5'-GACTCGAGTCGACATCGAT(17) -3' in order to reverse transcribe mRNA and obtain cDNA with an adapted sequence in the 3' end compatible to perform 3'-RACE-PCR. All clones were sequenced (Microsynth AG, Balgach, Switzerland). The open reading frame cDNA of rat SRP-27 digested with EcoRI and XhoI was used as probe to screen a mouse skeletal muscle UNI-ZAP XR cDNA library as previously described [16]. A clone of $2123 \mathrm{bp}$, containing the entire ORF was pulled out and sequenced (Microsynth AG, Balgach, Switzerland)

\section{Immunohistochemistry}

Cryosections of mouse diaphragm muscle were immunostained as described in Flucher et al. [22]. Primary antibodies and working dilutions were: affinity-purified rabbit ant-SRP-27 (see below; 1:100); affinity-purified rabbit anti-RyR1 at (1:2,000)[22]; mouse monoclonal anti-panRyR (C-34, Alexis Co., Lausen, Switzerland; 1:1,000); mouse monoclonal anti-SERCA2a (MA3-911; Affinity Bio Reagents, Neshanic Station, NJ; 1:1,000); mouse monoclonal antibody against $\alpha$ actinin (1:1,000). Secondary antibodies: Alexa488- and Alexa595-conjugated goat-anti-rabbit and goat-anti-mouse IgG (Molecular Probes, Eugene, OR) used at dilutions of 1:4,000. Images were recorded on a Zeiss Axiophot microscope with a cooled CCD camera and METAVUE imageprocessing software (Universal Imaging, West Chester, PA). 


\section{Western blots staining}

SDS-PAG electrophoresis and indirect immunoenzymatic staining were carried out as previously described [19]. Protein concentration was determined with the DC Protein Assay kit (Biorad) following the instruction provided by the manufacturer using bovine serum albumin as standard.

Polyclonal antibody production. Details are described in the Supplementary figure 1.

\section{Subcellular fraction preparation.}

Total microsomes from different rat tissues (heart, lung, brain, kidney, skeletal muscle, liver, spleen, stomach, intestine) were prepared as previously described [16,19]. Rat skeletal muscle SR fractions were obtained as previously described [23].

\section{Expression in COS-7 and TSA cells}

COS-7 cells were transfected with the SPR-27-pEYFP-N1 plasmid or the pEYFP-N1 plasmid as previously described [19]. Transfected COS-7 cells were imaged $48 \mathrm{~h}$ after transfection with a Nikon Eclipse TE 2000-U inverted fluorescent microscope equipped with a 100x Plan-Apo oil immersion objective and using a D1 digital camera (Nikon Inc). TSA cells were transfected with the SRP-27-pEYFP-N1 plasmid or the pEYFP-N1 plasmid as control using calcium phosphate as previously described [19]. Cells were used $48 \mathrm{~h}$ after transfection and the total microsomal fraction was prepared as described [19].

\section{Trypsin digestion}

Fifteen micrograms of protein were digested with increasing concentrations of trypsin, for 2 minutes at room temperature. The reaction was blocked by adding soybean trypsin inhibitor and samples were analyzed by staining western blot with anti-GFP Ab BD-Living Colors A.v. Peptide Ab (Clontech) or anti-calreticulin Ab (Santa-Cruz).

\section{Sucrose gradient}

Vesicles derived from the rat skeletal muscle membrane heavy (R4) fractions were solubilized in the presence of 1 or $0.2 \mathrm{M} \mathrm{NaCl}, 1 \%$ CHAPS, $0.003 \%$ egg phosphatidylcholine (L- $\alpha$ Lecithin, Egg Yolk), $10 \mathrm{mM}$ HEPES pH 7.4, $1 \mathrm{mM}$ DTT and anti-protease, for 30 minutes at $4^{\circ} \mathrm{C}$. Unsolubilized proteins were removed by centrifugation and soluble proteins were separated on a 5- 
$20 \%$ sucrose gradient by centrifugation at 25,000 rpm for $16 \mathrm{~h}$ in a SW40 Ti Beckmann rotor [11]. Fractions were collected and analyzed by SDS- PAG and immunoblot with anti-SRP27 Ab,

\section{Pull-down and co-immunoprecipitation experiments}

Proteins solubilized from the heavy SR R4 fractions (see above) were incubated with streptavidin polystyrene beads (Dynabeads M-280 Streptavidin, Dynal-Biotech) coated with biotinylated maurocalcine (40 $\mu \mathrm{M})$ in PBS in the presence of anti-proteases and BSA $(0.1 \mathrm{mg} / \mathrm{ml})$. After incubation, the beads were collected, washed and the bound proteins were analyzed either by immunoblot or autoradiography.

For co-immunoprecipitation experiments $1 \mathrm{mg}$ of rat skeletal muscle heavy SR was solubilized $\left(4^{\circ} \mathrm{C}, 30 \mathrm{~min}\right)$ in a buffer containing $1 \%$ CHAPS, $0.2 \mathrm{M} \mathrm{NaCl}, 10 \mathrm{mM}$ HEPES pH 7.4, 0.003\% lipids (L- $\alpha$-Lecithin, Egg Yolk), $1 \mathrm{mM}$ DTT and protease inhibitor cocktail in a final volume of $1 \mathrm{ml}$. After centrifugation (30 min 100,000xg) solubilized proteins were diluted 10 times with 0.2M NaCl, HEPES $10 \mathrm{mM}$ pH 7.4 and protease inhibitor cocktail and incubated for 2 hours at room temperature with ProteinA-Sepharose beads coated with anti-SRP-27 antibody (50 $\mu \mathrm{g}$ ) or Protein-G-Sepharose beads coated with anti-RyR antibody $(10 \mu g)$. Beads were then washed three times with PBS and the bound proteins were eluted with cracking buffer (6 M urea, $10 \mathrm{mM}$ SodiumPhosphate pH 7.2, 1 \% SDS, $1 \%$ ß-mercaptoethanol). Proteins present in the void, last wash and bound to the beads were separated by SDS PAG and blotted onto nitrocellulose.

\section{In vitro transcription.}

In vitro transcription [24] was performed using the TnT quick coupled transcription/translation system (Promega, Madison, WI, U.S.A.) according to the manufacturer instructions.

\section{Statistical analysis and Software}

Statistical analysis was performed using the Sudent's $t$ test for unpaired samples; means were considered statistically significant when the $\mathrm{P}$ value was $<0.05$. Images were processed using Gimp 2.2.13, Corel Photopaint 6.0 software. Blast alignments were performed on the NCBI web site using BLAST 2.2.8 released and highest. Multiple sequence alignments were performed using the Clustal W algorithm available from the Swiss node of the European Molecular Biology Network. Transmembrane domain prediction was obtained using TMhMM 2.0 on the Biology Workbench web site [25, 26]. 


\section{Results and Discussion}

The biochemical and molecular characterization of the proteins present in SR membrane is an important task for understanding the mechanisms underlying $\mathrm{Ca}^{2+}$ homoeostasis in skeletal muscle under normal and pathological conditions. To identify novel proteins of the skeletal muscle SR involved in E-C coupling we performed mass spectrometry analysis of the protein constituents of the junctional face membrane. Proteins of the junctional face membrane were separated on a SDS-PAGE and the bands corresponding to polypeptides having a molecular mass ranging from 10 to $100 \mathrm{kDa}$ were processed for electronspray mass spectrometry analysis. This approach allowed us to identify a 19 amino acid long peptide sequence from a band migrating as a single polypeptide chain of $27 \mathrm{kDa}$ (Fig.1, Rabbit pep). Its primary sequence matched that of a hypothetical protein in the NCBI database. We named this novel SR protein SRP-27, for sarcoplasmic reticulum $27 \mathrm{kDa}$ protein. EST encoding the predicted primary acid sequence of SPR-27 were then used to design PCR primers and to amplify a cDNA fragment from total rat mRNA which was used to i) screen a mouse skeletal muscle cDNA library and ii) to produce a recombinant fusion protein to be used as antigen to generate polyclonal antibodies against the COOH-terminal domain of SRP-27.

\section{cDNA cloning, sequence analysis and membrane topology of SRP-27.}

We isolated the full length SPR-27 rat cDNA and compared the deduced amino acid sequence with the peptide sequence obtained from the protein sequence of the rabbit skeletal muscle junctional face membrane (Fig.1). The rat cDNA contains an open reading frame of 891 bp corresponding to a 297 amino acid long polypeptide (rattus norvegicus NCBI accession number EF690436). The deduced primary sequence of mouse skeletal muscle SRP-27 (mus musculus NCBI accession number EF988666) predicts a protein of 298 amino acids with a molecular mass of 33 $\mathrm{kDa}$. The predicted $\mathrm{NH}_{2}$-terminal sequences of both rat and mouse SPR-27 are $84 \%$ identical to amino acid peptide sequence obtained from the $27 \mathrm{kDa}$ protein of the rabbit junctional face membrane. Multiple sequence alignments reveal that the mouse skeletal muscle cDNA clone encodes a protein displaying 97\% and 98\% identity with rat and human SRP-27 (Fig. 1). Transmembrane domain prediction (TMhMM) reveals four putative transmembrane segments.

Expression of SPR-27/pYEFP fusion protein in COS-7 cells revealed two patterns of subcellular distribution: in approx. 40\% of the transfected cells (437/1026) the fluorescence pattern was typically localized to the endoplasmic reticulum, as revealed by the reticular fluorescence originating around the nuclear membrane and extending into the cytoplasm. While 57\% (588/1026) of the transfected cells displayed large cisternae whose membranes are enriched in the YFP-tagged 
SPR-27 (Fig. 2A, 2B). The exact nature of the stained cisternae is unknown; these structures may represent dilations of ER membrane induced by the over-expression of SRP-27.

In order to determine if native SRP-27 present in skeletal muscle is an intrinsic membrane protein, we extracted SR membranes with $\mathrm{Na}_{2} \mathrm{CO}_{3}$ at $\mathrm{pH}$ 11. Figure 2C shows that after this treatment, SRP-27 is found in the insoluble membrane fraction indicating that it is an integral protein of sarco(endo)plasmic reticulum membranes. Interestingly, we also noticed that SRP-27 tends to form homo-oligomers as indicated by the immunopositive band of approx. $55 \mathrm{kDa}$ visible in figure $2 \mathrm{C}$ lane 2. The immunospecificity of this band was verified by pre-adsorption of SRP-27 Ab with the GST-SRP-27 fusion protein. Under these conditions both the upper and lower bands disappear (not shown).

The membrane topology of the $\mathrm{COOH}$ terminal domain of the expressed SRP-27-YFP protein was established by mild trypsin proteolytic treatment of the total microsomal fraction isolated from transfected TSA cells. Western blot staining with polyclonal anti-GFP Ab reveals that the $55 \mathrm{kDa}$ recombinant SRP-27-YFP fusion protein is degraded at increasing trypsin concentrations (Fig 2D, left). In control experiments carried out under similar conditions, the immunoreactivity of YFP was destroyed [27] (Fig.2D, right), whereas the reactivity of the intralumenal protein calreticulin was unaltered (Fig. 2D, lower panel), indicating that trypsin was excluded from the lumenal space. Thus, since the COOH terminal region of SRP-27 is degraded after trypsin digestion, it must be facing the cytoplasmic side of the endo(sarco)plasmic reticulum membrane.

\section{Tissue distribution and developmental expression of SRP-27.}

The tissue distribution of SRP-27 was investigated by western blot staining of proteins present in the microsomal fractions of different rat tissues, using polyclonal anti-SRP-27 Ab. Figure 3A shows that the SRP-27 kDa immunoreactive band is found in total rat microsomal membranes isolated from excitable tissues such as heart (lane 1), brain (lane 3) and skeletal muscle (lane 5) but is absent from lung, kidney, spleen, liver, stomach and intestine.

We also determined the pattern of developmental expression of SPR-27 in skeletal muscles. Its expression appears to be dependent on both the developmental stage and on the muscle fiber type. Indeed, the content of SRP-27 in the total miscrosomal fraction of skeletal muscles increases at day 15 and peaks at approximately 1-2 months of post-natal development (Fig. 3B, 3C). Furthermore, SRP-27 expression is 5 to 6 fold higher in fast twitch muscles compared to slow twitch muscles (Fig.3D, 3E). The increase in the SRP-27 content during post-natal development and its fiber type specific expression, suggest that this protein may be important in the post-natal maturation of skeletal muscle membranes. Since the SR is morphologically and functionally sub-specialized, we 
analyzed more precisely the subcellular distribution of SRP-27 within the SR membrane by a combination of biochemical and immunohistochemical techniques.

\section{Subcellular distribution of SPR-27}

Immunonocytochemistry (Fig. 4A and 4B) of muscle fibers from adult mouse diaphragm indicate that SPR-27 is highly concentrated in the perinuclear ER region (arrows) and to a lesser extent is localized throughout the muscle fibers. The distribution pattern of SRP-27 between the myofibrils is consistent with its localization in the SR. However, direct comparison of its staining pattern with that of the RyR1 indicates that the sarcomeric SRP-27 overlaps, but does not precisely co-localize with the RyR1. Double labeling of SRP-27, RyR1, or SERCA with $\alpha$-actinin show that the band of the SRP-27-containing compartment is located over the I-band. There it partially overlaps with the RyR1-labeled triads and alternates with the dominant SERCA band corresponding to longitudinal SR at the A-band. Thus, SRP-27 appears to also localize in a separate subcompartment of the SR, which is adjacent to, but distinct from that containing RyR or SERCA. Corroborating observations were made by double immunofluorescence labeling of SRP-27 with RyR in cultured mouse myotubes (supplementary Fig. 2). Whereas RyR1 was localized predominantly in clusters in the periphery of myotubes, SRP-27 was found in a membrane system throughout the myotubes. In addition, it accumulated between the nuclei in the center of the myotubes and in the nuclear envelope, indicating high concentrations of SRP-27 are also in the ER.

Western blot staining of SR fractions confirm data obtained by immunohistochemistry. SRP27 is distributed in light (R2) and heavy (R4) SR membrane fractions enriched in longitudinal SR membrane and in terminal cisternae (R4), respectively (Fig. 4C). The presence of SRP-27 around the $\mathrm{Z}$ line and in the membrane fractions corresponding to longitudinal SR and terminal cisternae, suggests that SRP-27 might be part of a macromolecular $\mathrm{Ca}^{2+}$ signaling complex involved in pumping $\mathrm{Ca}^{2+}$ back into the SR and/or in releasing it via the $\mathrm{Ca}^{2+}$ release channel to activate muscle contraction. To evaluate whether SRP-27 is involved in any aspect of SR $\mathrm{Ca}^{2+}$ handling, we analyzed its distribution after separation of the SR membrane proteins by sucrose density centrifugation. Terminal cisternae were solubilized in the presence of CHAPS (1\%) and $\mathrm{NaCl}(1 \mathrm{M})$ and the solubilized proteins separated on a continuous sucrose gradient (5 to 20\%). Western blot staining of the proteins from different fractions of the sucrose gradient with SRP-27 Ab (Figure 5 lower panel) shows that an important fraction of SRP-27 migrates in an intermediate density fraction containing other major SR proteins such as SERCA and calsequestrin (Fig. 5 upper panel, CBB stained gel). Interestingly, a remarkable fraction of the SRP-27 is also found in the sucrose density gradient fractions enriched in RyR (asterisk). The distribution of SRP-27 in the density gradient was 
found to depend on the ionic strength of the sucrose gradient buffer. At low ionic strength (200 mM $\mathrm{NaCl}$ ) the content of SRP-27 in the intermediate density fraction (enriched in SERCA and calsequestrin) is negligible and the bulk of SRP-27 is found in the fractions corresponding to a sucrose concentration of 15-18\%, which also contain the RyR complex (not shown). The presence of SRP-27 in these heavy sucrose fractions could result from the aggregation of SRP-27 leading to the formation of large complexes that co-sediment with the RyR complex. However, we think that it is unlikely since aggregation of at least $100 \mathrm{SRP}-27$ molecules would be required to form a $2.5 \mathrm{MDa}$ complex able to co-migrate with the RyR complex. On the other hand, the presence of SRP-27 in the fraction enriched in RyR after solubilization in 1\% CHAPS at low ionic strength, may suggest the existence of a macromolecular complex resulting from the direct or indirect association of SRP-27 with the RyR complex. In order to assess the existence of such an association, we performed two sets of experiments; in the first, solubilized SR proteins were pulled down using beads coated with maurocalcine, a specific effector of RyR. As shown in figure 6A the RyR was found to interact with maurocalcine coated beads. In addition, maurocalcine coated beads also pulled-down SRP-27 (Fig. 6A, right panel). Interestingly, SRP-27 obtained by in vitro translation was not pulled-down by maurocalcine coated beads (Figure 6B) indicating that SRP27 must bind to maurocalcine beads via another protein, most likely the RyR. In order to confirm that SRP-27 is part of a macromolecular complex containing the RyR, we performed co-immunoprecipitation experiments using anti-SRP27 (Fig. 6C, left panel) and anti- RyR antibodies (Fig. 6C right panel) as bait. To detect the interacting molecule, the western blots of the proteins bound to Sepharose-Prot. A beads were stained with anti-RyR (Fig. 6C, left panel) and with anti-SRP 27 (Fig. 6C right panel) antibodies. As shown in Fig 6C, the complementary co-immunoprecipitation experiments clearly reveal a macromolecular complex formed by both SRP-27 and the RyR.

In conclusion our results show that SRP-27 is widely distributed through muscle SR and ER, however, it also associates with the RyR, and thus is part of the sarcoplasmic reticulum macromolecular signaling complex involved in skeletal muscle EC coupling. 


\section{Acknowledgements}

The technical assistance of Malies Angebrand is gratefully acknowledged. This work was supported by grants from Telethon GGP05025, Ministero della Ricerca Scientifica e Tecnologica ex40\% e 60\%, Department of Anesthesia Basel University Hospital, HPRN-CT-2002-00331 from the European Union, and a grant from the Austrian Science Fund (P16532-B05) 


\section{References}

1. Ríos, E. and Pizarro, G. (1991) Voltage sensor of excitation-contraction coupling in skeletal muscle. Physiol. Rev. 71, 849-908.

2. Melzer, W., Herrmann-Frank, A., Lüttgau, H.C. (1995) The role of $\mathrm{Ca}^{2+}$ ions in excitationcontraction coupling of skeletal muscle fibres. Biochim. Biophys. Acta. 1241, 59-116.

3. Schneider, M.F. and Chandler, W.K. (1973) Voltage dependent charge movement of skeletal muscle: a possible step in excitation-contraction coupling. Nature 242, 244-246.

4. Franzini-Armstrong, C. A. and Jorgensen, A. O. (1994) Structure and development of E-C coupling units in skeletal muscle. Annu. Rev. Physiol. 56, 509-534.

5. Nakai, J., Dirksen, R. T., Nguyen, H. T., Pessah, I. N., Beam, K. G. and Allen, P. D. (1996) Enhanced dihydropyridine receptor channel activity in the presence of ryanodine receptor. Nature 380, 72-75.

6. Nakai, J., Sekiguchi, N., Rando, T. A., Allen, P. D. and Beam, K. G. (1998) Two regions of the ryanodine receptor involved in coupling with L-type $\mathrm{Ca}^{2+}$ channels. J. Biol. Chem. 273, 1340313406.

7. Adams, B.A., Tanabe, T., Mikami, A., Numa, S. and Beam, K.G. (1990) Intramembrane charge movement restored in dysgenic skeletal muscle by injection of dihydropyridine receptor cDNAs. Nature 346, 569-572.

8. Lamb, G.D. ( 2002) Voltage-sensor control of $\mathrm{Ca}^{2+}$ release in skeletal muscle: insights from skinned fibers. Front.Biosci. 7, 834-842.

9. Hamilton S.L. (2005) Ryanodine receptors. Cell Calcium 38, 253-260.

10. Paolini, C., Protasi, F. and Franzini-Armstrong, C. (2004) The relative position of RyR feet and DHPR tetrads in skeletal muscle. J. Mol Biol. 342,145-53.

11. Lai, F.A. Erickson, H.P., Rousseau, E., Liu, Q. Y. and Meissner, G. (1988) Purification and reconstitution of the calcium release channel from skeletal muscle. Nature 331, 315-319.

12. Kim, K.C., Caswell, A.H., Talvenheimo, J.A. and Brandt, N.R. (1990) Isolation of a terminal cisterna protein which may link the dihydropyridine receptor to the junctional foot protein in skeletal muscle. Biochemistry 29, 9281-9289.

13. Damiani, E., Tobaldin, G., Bortoloso, E. and Margreth, A. (1997) Functional behaviour of the ryanodine receptor/ $\mathrm{Ca}^{2+}$-release channel in vesiculated derivatives of the junctional membrane of terminal cisternae of rabbit fast muscle sarcoplasmic reticulum. Cell Calcium 22, 129-150. 
14. Zhang, L., Kelley, J., Schmeisser, G., Kobayashi, Y.M. and Jones, L.R. (1997) Complex formation between junctin, triadin, calsequestrin, and the ryanodine receptor. Proteins of the cardiac junctional sarcoplasmic reticulum membrane. J. Biol. Chem. 272, 23389-23397.

15. MacLennan, D.H. and Wong, P.T. (1971) Isolation of a calcium-sequestering protein from sarcoplasmic reticulum. Proc. Natl. Acad. Sci. U.S.A. 68, 1231-1235.

16. Anderson, A.A., Treves, S., Biral, D., Betto, R., Sandonà, D., Ronjat, M. and Zorzato, F. (2003) The novel skeletal muscle sarcoplasmic reticulum JP-45 protein. Molecular cloning, tissue distribution, developmental expression, and interaction with alpha 1.1 subunit of the voltage-gated calcium channel. J. Biol. Chem. 278, 39987-39992.

17. Takeshima, H., Komazaki, S., Nishi, M., Iino, M. and Kangawa, K. (2002) Junctophilins: a novel family of junctional membrane complex proteins. Mol. Cell 6, 11-22.

18. Nishi, M., Komazaki, S., Kurebayashi, N., Ogawa, Y., Noda, T., Iino, M. and Takeshima, H. (1999) Abnormal features in skeletal muscle from mice lacking mitsugumin29. J. Cell Biol. 147, 1473-1480.

19. Treves, S., Feriotto, G., Moccagatta, L., Gambari, R. and Zorzato F. (2000) Molecular cloning, expression, functional characterization, chromosomal localization, and gene structure of junctate, a novel integral calcium binding protein of sarco(endo)plasmic reticulum membrane. J. Biol. Chem. 275, 39555-39568

20. Kawasaki, T. and Kasai, M. (1994) Regulation of calcium channel in sarcoplasmic reticulum by calsequestrin. Biochem. Biophys. Res. Commun. 199, 1120-1127.

21. Murray, B. E. and Ohlendieck, K. (1997) Cross-linking analysis of the ryanodine receptor and alpha1-dihydropyridine receptor in rabbit skeletal muscle triads. Biochem. J. 324, 689-696.

22. Flucher, B. E., Conti, A., Takeshima, H. and Sorrentino, V. (1999) Type 3 and type 1 ryanodine receptors are localized in triads of the same mammalian skeletal muscle fibers. J. Cell Biol. 146, 621-30.

23. Saito, A., Seiler, S., Chu, A. and Fleischer, S. (1984) Preparation and morphology of sarcoplasmic reticulum terminal cisternae from rabbit skeletal muscle J. Cell. Biol. 99, 875-885.

24. Sandford, J, Codina, J. and Birnbaumer, L. (1991) Gamma-subunits of G proteins, but not their alpha- or beta-subunits, are polyisoprenylated. Studies on post-translational modifications using in vitro translation with rabbit reticulocyte lysates. J. Biol. Chem. 266, 570-9579.

25. Thompson, J.D., Gibson, T.J., Plewniak, F., Jeanmougin, F. and Higgins, D.G. (1997) The CLUSTAL_X windows interface: flexible strategies for multiple sequence alignment aided by quality analysis tools. Nucl. Acids Res. 25, 4876-4882. 
26. Blom, N., Gammeltoft, S. and Brunak, S. (1999) Sequence and structure-based prediction of eukaryotic protein phosphorylation sites. J. Mol. Biol. 294, 1351-1362.

27. Lorenz, H., Hailey, D.W. and Lippincott-Schwartz, J (2006) Fluorescence protease protection of GFP chimeras to reveal protein topology and subcellular localization. Nature Methods 3, 205-210. 


\section{Legends to Figures}

Figure 1. Predicted primary sequence of mouse SRP-27 and comparison with rat and human sequences. Black characters in grey boxes indicate conserved residues, white characters in dark grey boxes indicate non conserved residues. Putative transmembrane domains (TMhMM algorithm) are underlined in the mouse and rat sequences. Bold characters indicate the mass spec peptide sequence of rabbit junctional face membrane SRP-27.

Figure 2. SRP-27 is targeted to ER/SR membranes. Panels $A$ and B: COS-7 cells transfected with SRP-27-pEYFP-N1 cDNA show stack-like fluorescent bodies (A) or reticular fluorescence irradiating from the perinuclear region (B) (Bar $10 \mu \mathrm{m})$. Panel C: Microsomes from rat skeletal muscle (lanes 3 and 6) were treated with $100 \mathrm{mM} \mathrm{Na}_{2} \mathrm{CO}_{3} \mathrm{pH} 11$ to separate proteins loosely bound to membranes present in the supernatant (lanes 1 and 4) from integral membrane proteins (lanes 2 and 5) Ten micrograms of protein were separated on 10\% SDS-PAG and stained with Coomassie Brilliant Blue (lanes 1-3) or blotted onto nitrocellulose and probed with anti-SRP-27 antibody (lanes 4-6). Panel D: Crude microsomal fraction and the high speed supernatant were isolated from TSA cell transfected with SRP-27-YFP-N1 and the YFP constructs, respectively. $15 \mu$ g of protein were incubated with the indicated amounts of trypsin. After digestion, $11.25 \mu \mathrm{g}$ protein were separated on a 12\% SDS-PAG, blotted onto nitrocellulose and probed with anti-GFP antibodies. Lower panel shows the immunoreactivity with anti-calreticulin Ab, demonstrating that the vesicles were still intact after treatment with trypsin.

Figure 3. Expression of SRP-27 in different tissues and during development. Panel A microsomal proteins from the indicated tissues (10 $\mu \mathrm{g}$ of skeletal muscle, $60 \mu \mathrm{g}$ of all other tissues) were separated on a 10\%SDS-PAG, blotted onto nitrocellulose and probed with anti-SRP-27 antibody. Lane 1, heart; lane 2, lung; lane 3, brain; lane 4, kidney; lane 5, skeletal muscle; lane 6, liver; lane 7, spleen; lane 8, stomach; lane 9: intestine. Panels B and C: expression level of SRP-27 during post-natal development: $10 \mu \mathrm{g}$ of microsomal proteins obtained from rats of different ages (1day, 5 days; 10 days; 15 days; 1 month; 2 months) were separated on a $10 \%$ SDS-PAG blotted onto nitrocellulose and stained with anti-SRP-27 antibodies (panel B). Panel C shows the densitometric analysis of the immunopositive bands. Values were normalised to the signal obtained from 2 month old rats (100\%, bars represent mean \pm S.D., $n=3)$. Panel $\mathbf{D}$ and $\boldsymbol{E}$ : SRP-27 is enriched in fast-twitch muscles. Ten $\mu \mathrm{g}$ of microsomal proteins obtained from Soleus and EDL muscles were separated on a 12\% SDS-PAG blotted onto nitrocellulose and stained with anti-SRP-27 antibodies 
(panel D). Panel E shows the densitometry analysis. Values were normalised to the EDL signal (100\%, bars represent mean \pm S.D., $n=3)$.

\section{Figure 4. Subcellular localization of SRP-27 in the ER and SR of skeletal muscle fibers. Panel}

A: Double immunofluorescence labeling of SRP-27 (upper row and red in merged color image) and RyR1 (center row and green in merged color image) in mouse diaphragm muscle. SRP-27 is highly concentrated around myonuclei (arrows) and throughout the cytoplasm. In cross sections (left column) SRP-27 and RyR1 immunoreactivity is localized between the myofibrils (merged color image shows detail indicated above). In longitudinal sections (center and right columns) the SRP-27 labeling pattern forms fuzzy cross bands (arrowheads), which overlap, but are distinct from the double rows of triads labeled with the RyR1 ( $B$, fluorescent bead used for alignment of frames). Panel B: Double labeling of SRP-27, RyR1, or SERCA (upper row and green in the merged color image) with $\alpha$-actinin (center row and green in merged color image) in longitudinal sections of mouse diaphragm muscle. SRP-27 and the markers for junctional and longitudinal SR show clearly distinct sarcomeric banding patterns (compare the 2.5x magnified insets). The vesicular structures containing SRP-27 are localized along the I-bands, whereas RyR1 is found on either side of the Zline and the dominant SERCA band over the A-band. SRP-27 and RyR1 are found in all fibers, whereas slow muscle fibers $\left(^{*}\right)$ are not stained with the SERCA2a antibody. (Bar, 10 $\left.\mu \mathrm{m}\right)$. Panel C: Localisation of SRP-27 in sarcotubular membrane fractions: $10 \mu \mathrm{g}$ of protein from sarcotubular membrane fraction (R1-fraction enriched in sarcolemma, T tubules, longitudinal SR; R2-fraction enriched in longitudinal SR; R3-fraction containing a mixture of longitudinal SR and terminal cisternae; R4- terminal cisternae) were separated on a 12\% SDS-PAG, blotted onto nitrocellulose and stained with anti-SRP-27 antibodies. Images were processed using The Gimp 2.2.13

Figure 5. SRP-27 co-sediments with the RyR1 on a sucrose gradient. $1 \mathrm{mg}$ of protein from rat skeletal muscle terminal cisternae were solubilized with CHAPS $1 \%, 1 \mathrm{M} \mathrm{NaCl}$ as described in experimental procedures. Solubilized proteins were separated on a continuous sucrose gradient and fractions of 15 drops were collected. Forty five $\mu$ l of each fraction were separated on a 6\% (upper panel) or $12 \%$ (lower panel) SDS-PAG. Proteins were stained with Coomassie Brilliant Blue. (upper panel); arrows indicate CaATPase (SERCA), calsequestrin (CS) and the ryanodine receptor (RyR). The lower panel depicts the immunoblot stained with anti-SRP-27 antibodies. *shows fractions in which SRP-27 and the RyR co-sediment. 
Figure 6. SRP-27 is part of the RYR macromolecular complex. Panel A: heavy SR vesicles were solubilized with $1 \%$ CHAPS, $0.2 \mathrm{M} \mathrm{NaCl}$. The solubile proteins were incubated with beads coated with maurocalcine. Proteins present in the void (lane 1,3), last wash (lane 2,4) and bound to maurocalcine beads (lane 3,5) were stained with anti-RyR (left panel) and anti-SRP27 antibodies (right panel). Panel B: $\left[{ }^{35} \mathrm{~S}\right]$ labelled SRP-27 was obtained by in vitro translation. The labelled protein was incubated with beads coated with maurocalcine (left panel, $+\mathrm{M}$ ) or with an unrelated peptide (right panel, Crtl). Proteins present in the void (lane V), last wash (lane LW) and bound to beads (lane B) were separated on a 10\% SDS PAG SDS-PAG and detected by autoradiography. Panel C: co-immunorpecipitation experiments were performed on solubilized SR vesicles as described in the methods section. Left panel: solubilized SR proteins were incubated with protein-A Sepharose beads coated with anti-SRP-27 Ab; right panel: solubilized SR proteins were incubated with protein-G Sepahrose beads coated with anti-RyR Abs. Proteins present in the void (lane 1), last wash (lane 2) or bound to the beads (lane 3), were separated on a $6 \%$ SDS PAG (left) or $10 \%$ SDS PAG (right), blotted onto nitrocellulose and probed with the indicated antibody. 
Fig. 2
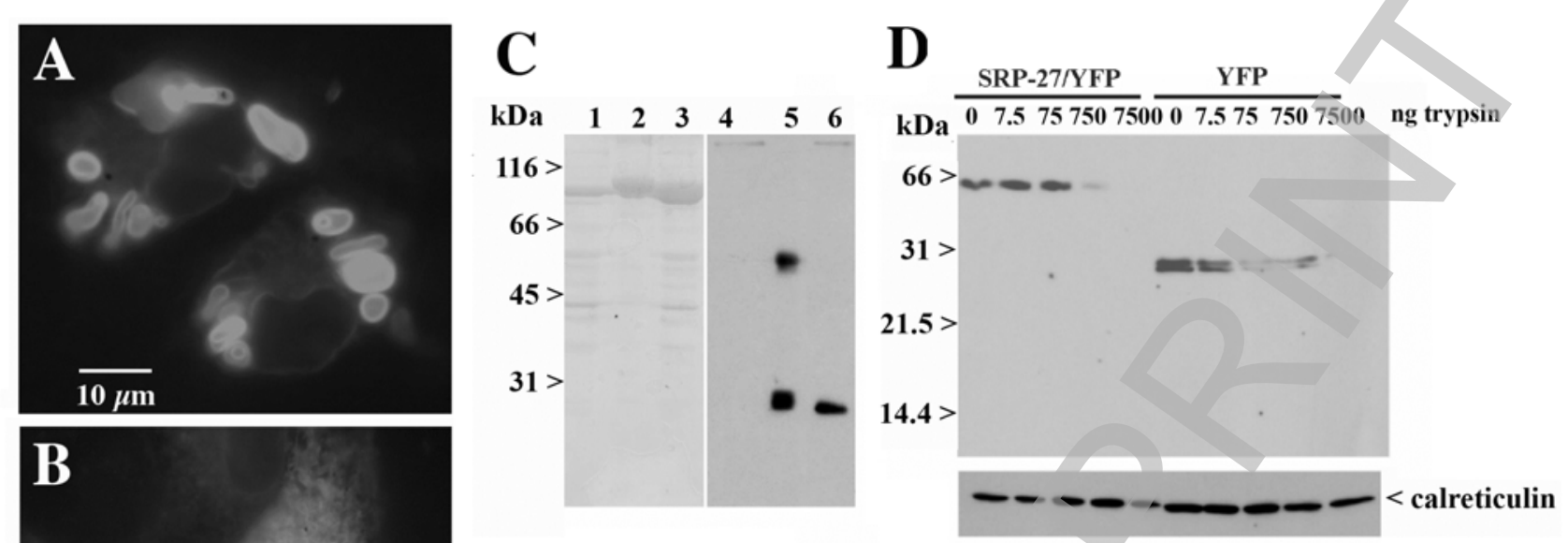

$10 \mu \mathrm{m}$

Licenced copy. Copying is not permitted, except with prior permission and as allowed by law. (C) 2007 The Authors Journal compilation (C) 2007 Biochemical Society 
Fig. 3
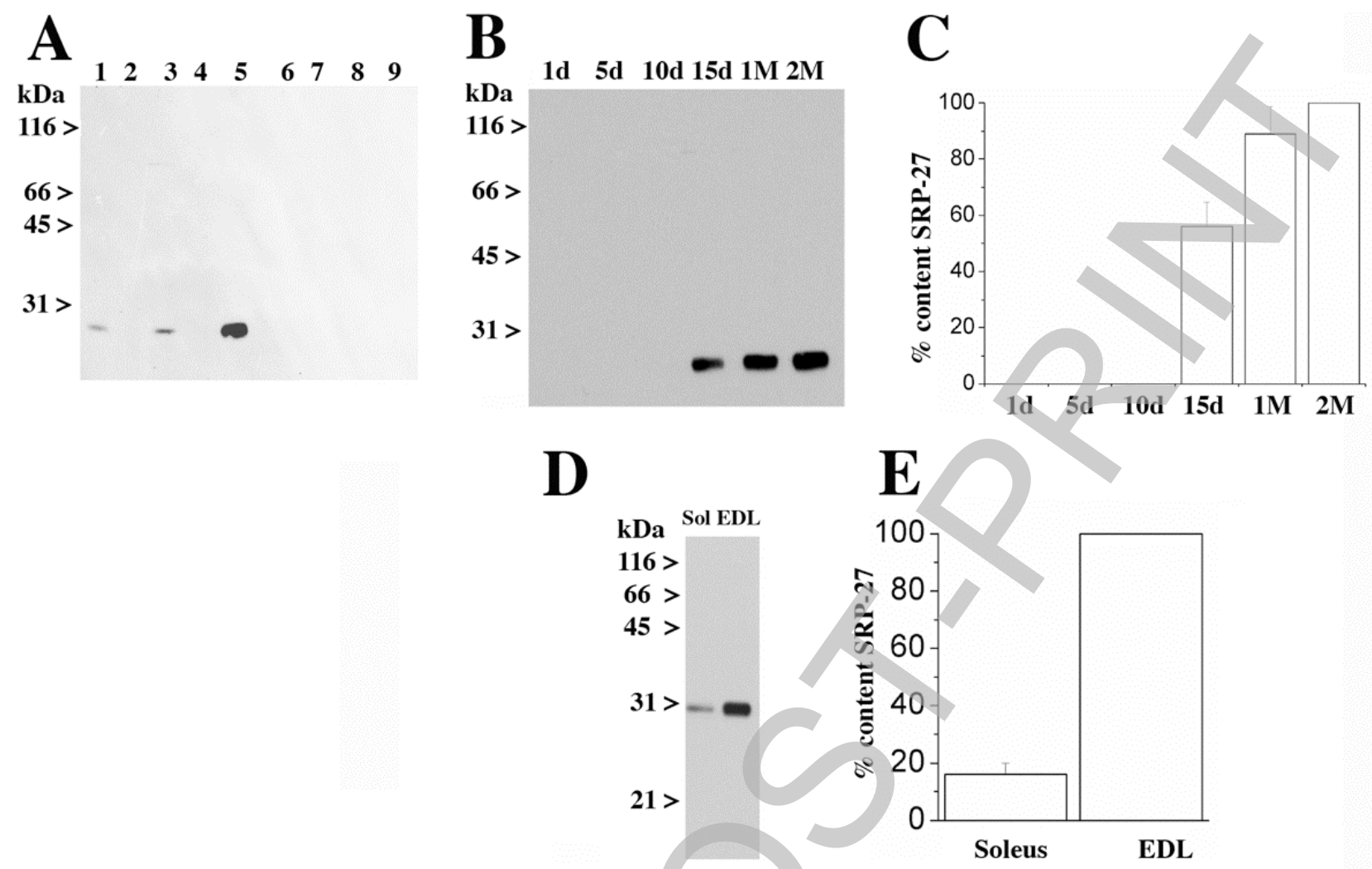
B Biochemical Journal Immediate Publication. Published on 26 Nov 2007 as manuscript BJ20070906

\section{1}

Fig. 4

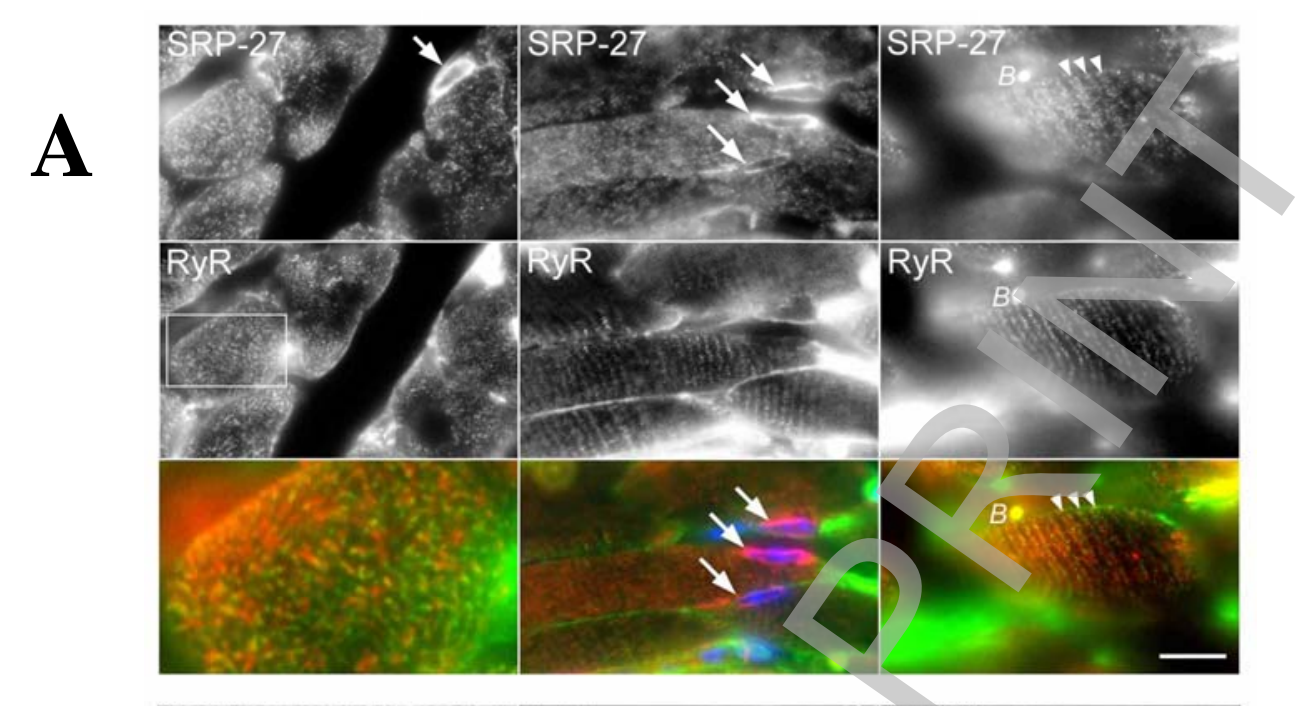

B

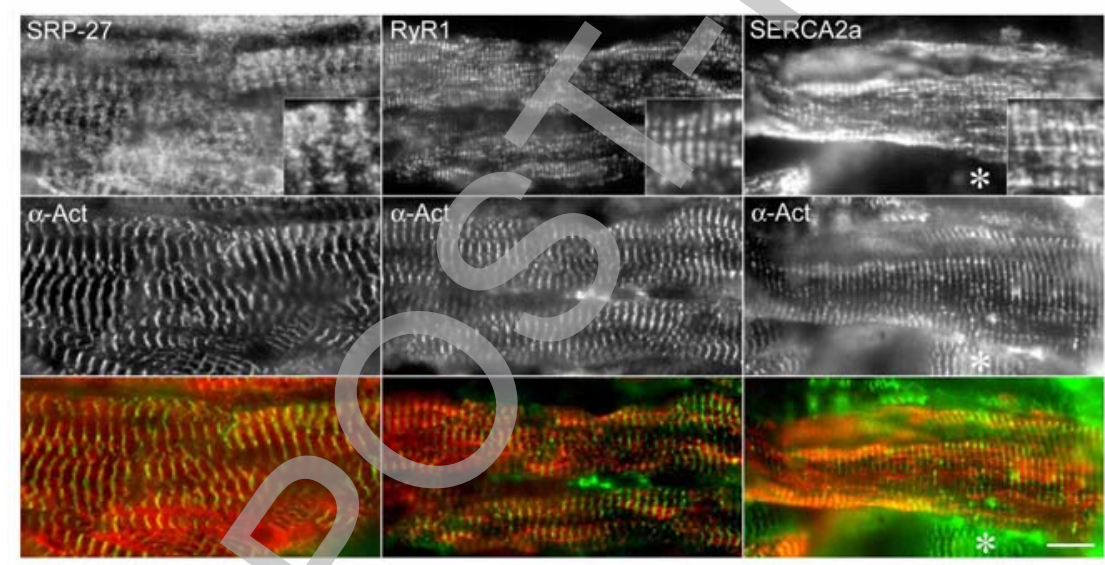


Fig. 5

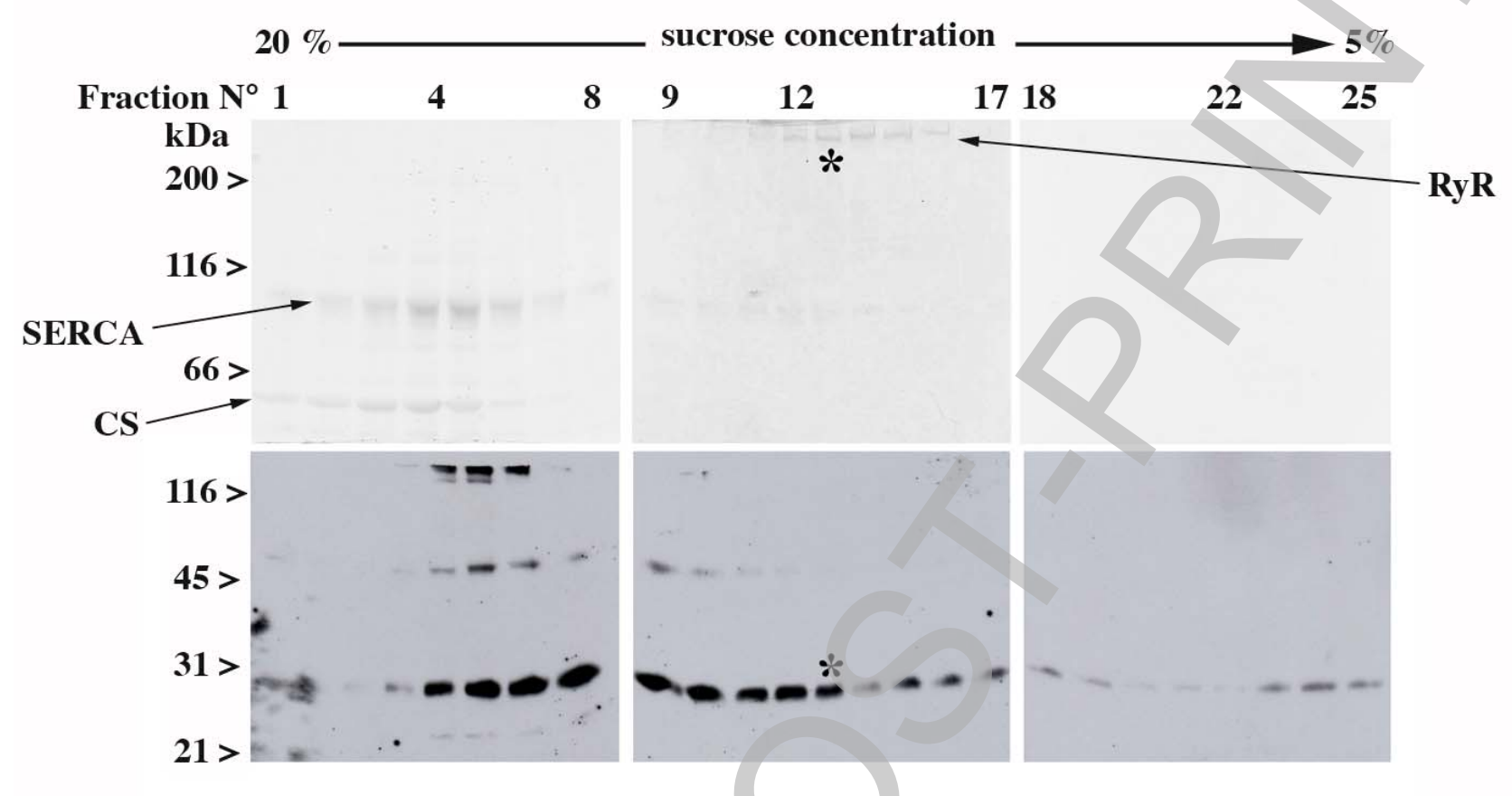

Licenced copy. Copying is not permitted, except with prior permission and as allowed by law. (C) 2007 The Authors Journal compilation (C) 2007 Biochemical Society 
Fig. 6
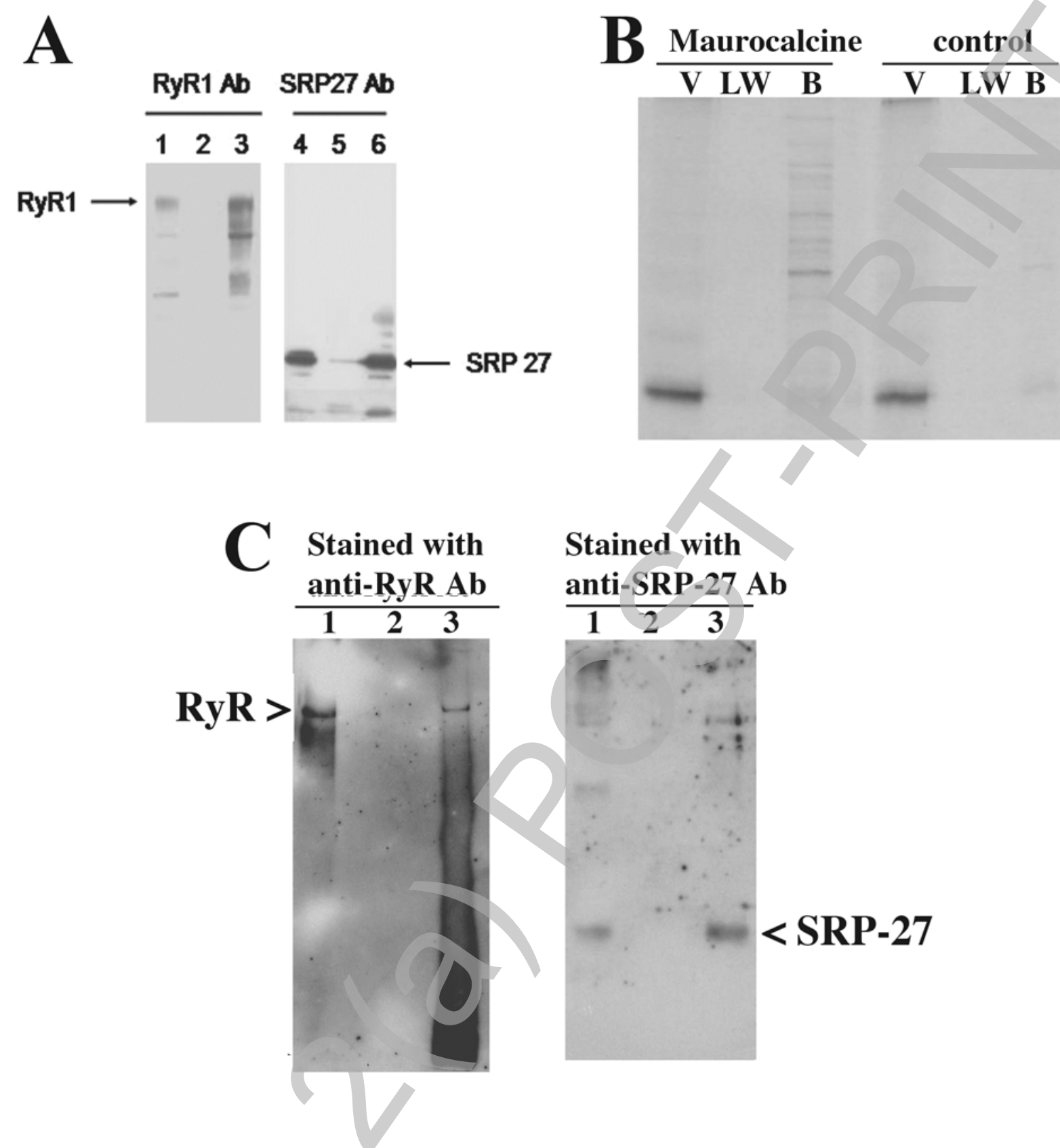